\title{
DESIGN OF AN EXPERIMENTAL DEVICE FOR THE MEASURE- MENT OF THE COEFFICIENT OF RESTITUTION
}

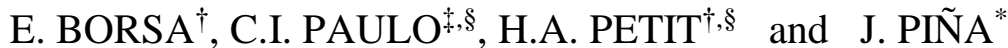 \\ $\dagger$ Departamento de Ciencias Básicas, Facultad de Ingeniería, Universidad Nacional del Centro de la Provincia \\ de Buenos Aires (UNCPBA). Av. Del Valle 5737 - 7400 Olavarría-Argentina. \\ \$ Departamento de Ingeniería Química, Facultad de Ingeniería, UNCPBA. Av. Del Valle 5737 - 7400 Olava- \\ rría-Argentina \\ $\S$ Centro de Investigaciones en Física e Ingeniería (CIFICEN), dependiente de UNCPBA-CICPBA-CONICET. \\ Av. Del Valle 5737 - 7400 Olavarría -Argentina \\ * Planta Piloto de Ingeniería Química (PLAPIQUI), dependiente de Universidad Nacional del Sur - CONI- \\ CET. Camino La Carrindanga km. 7 - 8000 Bahía Blanca - Argentina \\ eborsa@fio.unicen.edu.ar,cpaulo@fio.unicen.edu.ar,hpetit@fio.unicen.edu.ar,julianap@plapiqui.edu.ar
}

\begin{abstract}
In this work an experimental device was designed and built to measure the coefficient of restitution of particles of different types and sizes. The aim of the study is to analyze the dependence of the coefficient of restitution with the impact velocity, the shape and mass of the particles. Three types of particles of different origin were used: lentils, urea granules and polyethylene pellets. Two impact materials were evaluated: steel and polycarbonate. Image processing software was used to obtain the position of the particle at the instants before and after the impact, so the velocities and the coefficient of restitution of particles were obtained. For the 120 studied cases, the tangential values of the coefficient were always higher than the normal values. The highest values for the coefficient of restitution's modulus were found for the lower mass particles. Regarding the behavior on impact materials, steel was less elastic than polycarbonate.
\end{abstract}

Keywords - coefficient of restitution, particulate material, image processing.

\section{INTRODUCTION}

In recent years the technology of gas-solid separators has evolved to use them as dust classifiers and obtain particle separations with specific cut sizes, suitable for each industry requirement. The separation efficiency of the particulate material is related to operating variables of the process (concentration of solids in the feed, inlet velocity of the carrier gas, pressure drop, among others parameters), and to physical and morphological characteristics of the particles, mainly their size, shape and coefficient of restitution. Therefore, the processes of separation and classification of solids depend on both, macroscopic aspect (or process variables) and microscopic parameters related to the properties of the particles.

The coefficient of restitution (CoR) is a measure of the degree of conservation of kinetic energy in a particle-wall or particle-particle collision. It is a function of the velocity of the particle and can be calculated as the ratio between the velocity of the particle acquired after the impact and the velocity reached before the impact (Fernandez Llana, 2010). CoR is an important measure of energy dissipation in a wide variety of industrial processes that involve a solid material and a fluid that transports it (gas or liquid). The interactions between particles, particles and the carrier fluid and particles with the walls of the equipment, constitute aims of study in fluidization, granulation, agglomeration and separation particles processes. These interactions are in continuous study and not totally understood at present (Kotoky et al., 2018). On the other hand, the CoR represents one of the fundamental properties of the particles, necessary in simulations of computational fluid dynamic combined with the discrete element method (CFDDEM). The more accurate the input parameters for DEM, the more accurate the simulation will be (Liu et al., 2016). The same goes for other approaches, such as the Euler-Euler-CFD used for fluidized beds (Loha et al., 2014).

There is an extensive amount of experimental work on the measurement of the coefficient of restitution of spherical and large particles such as tennis balls and golf balls (Colombo et al., 2016; McNally et al., 2016; Espinosa et al., 2016). Large spherical particles require simple measurement techniques. However, at present it is a real challenge to accurately measure the coefficient of restitution for small and irregular particles. Crüger et al. (2016) measured the CoR of glass particles of about $1 \mathrm{~mm}$ in diameter on a wet surface, correlating the experimental values obtained with two theoretical models for the wet coefficient of restitution. Hastie (2013) measured the dry CoR for polyethylene pellets of around $4 \mathrm{~mm}$ in diameter, impacting on two different materials.

The objective of this work is to design and build an experimental device to accurately measure the average coefficient of restitution of small, irregular and different types of particles. The variables analyzed were the type of particle, the impact surface and the height from which each particle is thrown. The obtained results constitute an advance in the understanding of the dependence of the CoR with the microscopic studied variables related to particle characteristics. These values of CoR could be useful to improve mathematical prediction models and adjust the current numerical simulations of gas-solid dust classifiers. 


\section{MATERIALS AND METHODS}

\section{A. Materials}

Three types of particles of different origin and shape were used: lentils, urea granules and polyethylene pellets. Lentils are for commercial food use, urea granules are a commercial fertilizer and the pellets are commercial linear low-density polyethylene (LLDPE). The geometry of the urea granules is similar to small spheres (Fig. 1.a), while the lentils have an ellipsoidal shape (Fig. 1.b.1 and b.2) and the polyethylene pellets are small discs (Fig. 1.c.1 and c.2). The average mass of the urea particle was $0.0083 \mathrm{~g}$, the lentil particle presented an average mass of $0.0602 \mathrm{~g}$, while the mass of the polyethylene pellet was $0.0453 \mathrm{~g}$. For these measurements, an Alsep EX-2000A balance was used.

To analyze the shape and size of the particles, the ImageJ software was used, processing and analyzing the images obtained in 2D with a Motorola XT1540 13MP camera. The Feret diameter was used to characterize the particles by size. This diameter represents the distance between two parallel lines that are tangential to the contour of the projection of the particle. To classify them according to their shape, the circularity parameter (C) was used, which represents a measure of the approximation of the particles to a perfect circle (Takashimizu and Iiyoshi, 2016):

$$
C=\frac{4 \pi A}{P^{2}}
$$

where $A$ is the projected area and $P$ is the perimeter of the particle.

\section{B. Experimental Device}

The experimental device was designed in polycarbonate and consists of a structure with a closed rectangular prism shape, with an input through which the particles are introduced. The dimensions of the device are $60 \mathrm{~cm}$ wide, $40 \mathrm{~cm}$ high and $20 \mathrm{~cm}$ deep (Fig. 2). Each particle is dropped from a certain initial height without initial velocity (free fall). The particles were initially thrown from the different distances holding them with a metal clamp, starting from rest and in vertical position. Once the fall occurs, the particle impacts on a mobile surface inclined 45 degrees (Fig. 2).

The surface materials, on which the particle impacts, were of two different types: steel $3 \mathrm{~mm}$ thick and polycarbonate $2 \mathrm{~mm}$ thick.

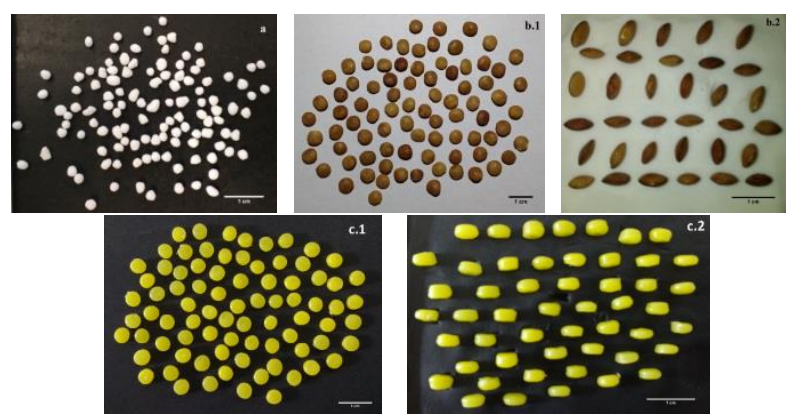

Fig. 1. a. Urea granules. b. Commercial lentil particles: b.1.Upper view; b.2. Side view. c. Commercial polyethylene pellets: c.1.Upper view; c.2. Side view.

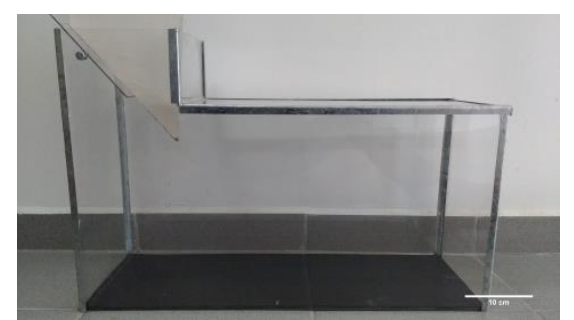

Fig. 2. Own design experimental device for CoR measurements, built in polycarbonate.

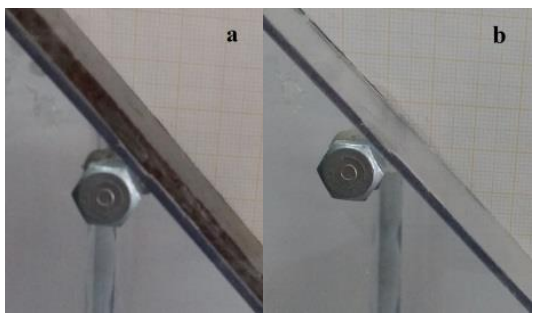

Fig. 3. Area of impact where the particle fall. Steel. b. Polycarbonate.

Figure 3 shows the area of impact where the particles fall, which constitutes the analysis sector of the device for the subsequent determination of the CoR. Behind the impact surface, a sheet of graph paper was placed to measure, through the captured images, the distance traveled by the particles during the impact and rebound.

\section{Capture and Processing of Images}

Using a high-resolution SJCAM3000 camera, the trajectories of the particles in the free fall and its impact on the inclined surface were filmed. The rotation of the particles during their free fall was not taken into account for this work. Results are reported for a resolution of $720 p$ and 120 frames per second (fps). The studied cases were also recorded with a lower number of frames per second, but the increase in the quality of the images did not allow capturing clearly the moment of the particle impact. To process the obtained videos, the VLC Media Player software was used, through which four frames were extracted per particle studied. Two of the frames correspond to the moment before the impact, and the other two immediately after the impact with the surface.

With the free software Image J $1.50 \mathrm{i}$, the obtained images were analyzed to determine the positions of the particle in each of the four selected frames. Considering these coordinates, the displacements in both axes $\Delta x$ (and $\Delta y$ ), before and after the impact, were calculated:

$$
\begin{aligned}
& \Delta x=x_{t+1}-x_{t} \\
& \Delta y=y_{t+1}-y_{t}
\end{aligned}
$$

Then, the displacement in module was calculated for both instants:

$$
d=\sqrt{(\Delta x)^{2}+(\Delta y)^{2}}
$$

Considering the time passed during a frame $\left(t_{f}=\right.$ $t+1-t)$ and the displacement in modulus reached before and after impact, $d_{b i}$ y $d_{a i}$ (Eq. 4), the corresponding velocities of the particle were calculated:

$$
V_{b i}=\frac{d_{b i}}{t_{f}}
$$




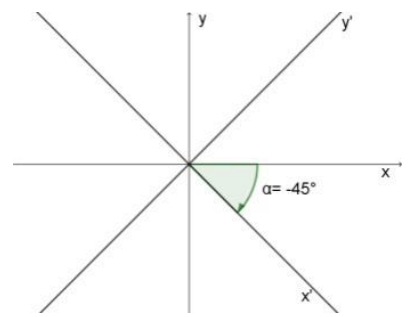

Fig. 4. Rotation of the reference system.

$$
V_{a i}=\frac{d_{a i}}{t_{f}}
$$

The coefficient of restitution is a measure of the elasticity between the bodies that impact, and it is between 0 for perfectly plastic collisions and 1 for perfectly elastic collisions. Considering the velocity before the impact $\left(V_{b i}\right)$ and the velocity after the impact $\left(V_{a i}\right)$ of two bodies "1" and "2" that collide, the $\operatorname{CoR}$ can be defined as (Hastie, 2013):

$$
\text { CoR }=\frac{\text { Vai2 }- \text { Vai1 }}{\text { Vbi1-Vbi2 }}
$$

If the particle is defined as the body " 1 ", and the impact surface as the body " 2 ", and it is also considered that this surface is fixed as in the experimental device of this work $\left(V_{b i 2}=V_{a i 2}=0\right)$, the module of the coefficient of restitution $\left(\operatorname{CoR}_{m}\right)$ is reduced to:

$$
C o R_{m}=\left|\frac{-V a i 1}{V b i 1}\right|=\left|\frac{-V a i}{V b i}\right|
$$

To calculate the normal coefficient of restitution $\left(C o R_{N}\right)$ and the tangential coefficient of restitution $\left(\operatorname{CoR}_{T}\right)$, the angle of inclination of the surface where the particle impacts is considered, and a rotation of the reference system is performed to find the new coordinates of the particle before and after the impact (Fig. 4). The new coordinates are:

$$
\left(\begin{array}{ll}
x^{\prime} & y^{\prime}
\end{array}\right)=\left(\begin{array}{ll}
x & y
\end{array}\right)\left(\begin{array}{cc}
\cos \alpha & -\operatorname{sen} \alpha \\
\operatorname{sen} \alpha & \cos \alpha
\end{array}\right)
$$

Considering the new coordinates, the tangential displacement $\left(\Delta x^{\prime}\right)$ and the normal displacement of the particles $\left(\Delta y^{\prime}\right)$ are calculated as previously (Eqs. 2 and 3):

$$
\begin{aligned}
& \Delta x^{\prime}=x_{t+1}^{\prime}-x_{t}^{\prime} \\
& \Delta y^{\prime}=y^{\prime}{ }_{t+1}-y_{t}^{\prime}
\end{aligned}
$$

Taking into account the time passed during a frame $\left(t_{f}=t+1-t\right)$ and the tangential displacement reached before and after the impact, $\Delta x_{b i}^{\prime}$ y $\Delta x_{a i}^{\prime}$ (Eq. $10)$, the corresponding tangential velocities of the particle were calculated:

$$
\begin{aligned}
& V_{T b i}=\frac{\Delta x_{b i}^{\prime}}{t_{f}} \\
& V_{T a i}=\frac{\Delta x_{a i}^{\prime}}{t_{f}}
\end{aligned}
$$

Likewise, the normal particle velocities were calculated:

$$
\begin{aligned}
& V_{N b i}=\frac{\Delta y_{b i}^{\prime}}{t_{f}} \\
& V_{N a i}=\frac{\Delta y_{a i}^{\prime}}{t_{f}}
\end{aligned}
$$

From the tangential and normal velocities, before and after the impact, the corresponding tangential and normal restitution coefficients were calculated $\left(\mathrm{CoR}_{T}\right.$ and $\left.\operatorname{CoR}_{N}\right)$ :

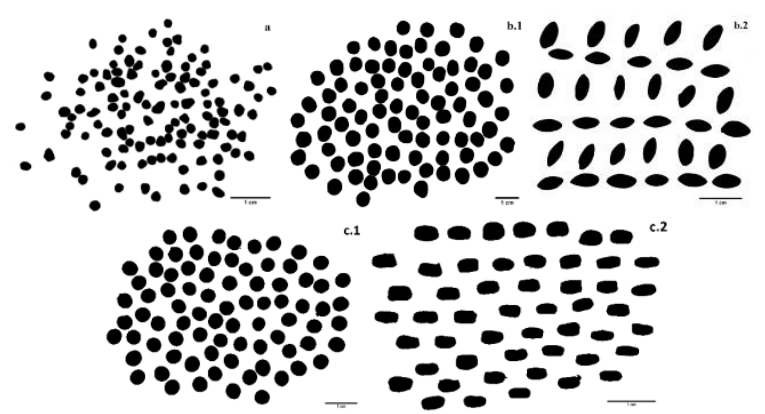

Fig. 5. Binary images of the particles studied. a. Urea granules. b.1. Lentils, upper view. b.2. Lentils, side view. c.1. Polyethylene pellets, upper view. c.2. Polyethylene pellets, side view.

$$
\begin{gathered}
\operatorname{CoR}_{T}=\left|\frac{-V_{T a i}}{V_{T b i}}\right|=\left|\frac{-\Delta x^{\prime} a i}{\Delta x_{b i}}\right| \\
C o R_{N}=\left|\frac{-V_{N a i}}{V_{N b i}}\right|=\left|\frac{-\Delta y^{\prime} a i}{\Delta y_{b i}^{\prime}}\right|
\end{gathered}
$$

\section{RESULTS AND DISCUSSION}

Figure 5 (a, b.1, b.2, c.1 y c.2) shows the binary images of the particles processed with ImageJ software.

Table 1 shows the average morphological characteristics corresponding to the analyzed particles. It is observed that the urea particles are smaller in size and mass than the lentils particles, but they have similar values of circularity, considering the upper view of the lentils.

Figure 6 shows a sequence of frames corresponding to the instants before and after the impact of a urea particle, thrown from $2 \mathrm{~cm}$, which impacts on the polycarbonate surface.

Tables 2 and 3 show the experimental results for the studied cases. The reported values are the average of 10 tests per case, so a total of 120 cases have been studied. Regarding the analysis of the launching heights, the particles thrown from $3 \mathrm{~cm}$ have greater velocity before the impact than the corresponding to $2 \mathrm{~cm}$ for all the analyzed cases. This behavior agrees with what is expected for particles accelerated in free fall.

Respect to the different impact materials, the coefficient of restitution $\left(\operatorname{CoR}_{m}\right)$ for the urea particles, that impact on polycarbonate surface, is greater than for those that collide with the steel plate in $10.7 \%$. The same behavior is observed for lentil particles and polyethylene pellets with an increase of $5.03 \%$ and $9.82 \%$ respectively (Table 2 ). In these cases, steel behaves as a less elastic material than polycarbonate.

The values of the coefficient of restitution for urea particles are higher than for polyethylene pellets and lentil particles in all the studied cases. The highest value was obtained for study case 1 ( $C o R_{m}$ : 0.718$)$, corresponding to urea particles on polycarbonate. This behavior was expected due to the higher elasticity of polycarbonate against steel, and because urea is the material with the least mass. Regarding the type of particle, the shape influences notably on the values obtained of $\mathrm{CoR}_{\mathrm{m}}$. Although the circularities for the top view of len- 
Table 1. Morphological characteristics of the particles studied.

\begin{tabular}{|c|c|c|c|c|}
\hline \multirow{2}{*}{ Particle } & \multirow{2}{*}{ Mass $[\mathrm{g}]$} & \multicolumn{2}{|c|}{ Feret's diameter $[\mathrm{cm}]$} & \multirow{2}{*}{ Circularity } \\
\hline & & Max. & Min. & \\
\hline Urea & 0.0083 & 0.273 & 0.229 & 0.874 \\
\hline \multirow{2}{*}{ Lentil } & \multirow{2}{*}{0.0602} & $0.666\left(\mathrm{UV}^{*}\right)$ & 0.588 (UV) & $0.894(\mathrm{UV})$ \\
\hline & & $0.644(\mathrm{SV} *)$ & 0.318 (SV) & $0.659(\mathrm{SV})$ \\
\hline \multirow{2}{*}{ Polyethylene } & \multirow{2}{*}{0.0453} & $0.481\left(\mathrm{UV}^{*}\right)$ & 0.440 (UV) & 0.874 (UV) \\
\hline & & 0.483 (SV*) & 0.275 (SV) & 0.608 (SV) \\
\hline
\end{tabular}

*UV: upper view. SV: side view.

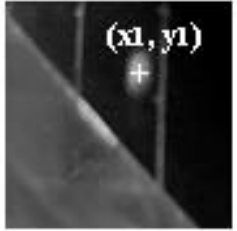

Position 1

Position 2

before the impact before the impact
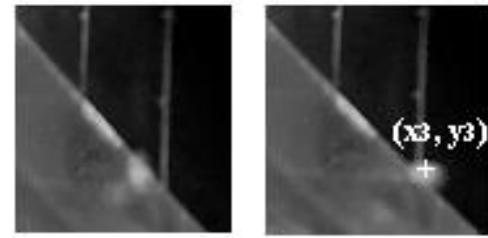

Position 3 after the impact

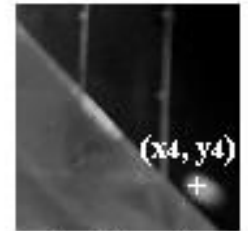

Position 4

after the impact

Fig. 6. Frames captured of a urea particle that impacts on polycarbonate surface.

Table 2. Experimental result for the cases studied.

\begin{tabular}{cllccccc}
\hline Cases & Particle & Impact surface & Launch height $[\mathrm{cm}]$ & $V_{b i}[\mathrm{~cm} / \mathrm{s}]$ & $V_{a i}[\mathrm{~cm} / \mathrm{s}]$ & $\operatorname{CoR}_{m}$ & $\operatorname{CoR}_{\text {mean }}$ \\
\hline 1 & Urea & Polycarbonate & 2 & 68.546 & 49.187 & 0.718 & 0.702 \\
2 & Urea & Polycarbonate & 3 & 81.509 & 55.885 & 0.687 & \\
3 & Urea & Steel & 2 & 82.667 & 54.982 & 0.667 & 0.634 \\
4 & Urea & Steel & 3 & 102.615 & 61.157 & 0.600 & \\
5 & Lentil & Polycarbonate & 2 & 68.761 & 46.377 & 0.678 & 0.626 \\
6 & Lentil & Polycarbonate & 3 & 80.982 & 46.061 & 0.573 & \\
7 & Lentil & Steel & 2 & 79.228 & 50.319 & 0.642 & 0.596 \\
8 & Lentil & Steel & 3 & 99.046 & 52.565 & 0.551 & \\
9 & Polyethylene & Polycarbonate & 2 & 70.659 & 49.642 & 0.704 & 0.671 \\
10 & Polyethylene & Polycarbonate & 3 & 91.390 & 58.155 & 0.638 & \\
11 & Polyethylene & Steel & 2 & 79.323 & 48.914 & 0.624 & 0.611 \\
12 & Polyethylene & Steel & 3 & 92.763 & 54.681 & 0.598 & \\
\hline
\end{tabular}

tils and polyethylene pellets are similar, for the side view the value of circularity drops markedly, due to their ellipsoidal and discs shapes. This particularity of the particles causes the $\operatorname{CoR}_{m}$ value to vary depending on the surface (upper or side) with which it impacts. Besides, $\mathrm{CoR}_{m}$ increases when the size of the particle decreases. These phenomena indicate a direct dependence of the $\operatorname{CoR}_{m}$ with the type, shape and size of the particle analyzed.

Table 3 shows that the values calculated for the normal coefficient of restitution are less than the tangential ones, in all the studied cases. Furthermore, the highest values are obtained for the urea particles colliding on polycarbonate, as observed for $\mathrm{CoR}_{m}$.

Table 3. $\mathrm{CoR}_{\mathrm{T}}$ and $\mathrm{CoR}_{\mathrm{N}}$ values obtain from studied cases.

\begin{tabular}{|c|c|c|c|c|c|c|c|}
\hline Cases & Particle & Impact surface & Launch height $[\mathrm{cm}]$ & $\operatorname{CoR} T$ & CoRTmean & $\operatorname{CoR}_{N}$ & $\operatorname{CoR}_{N}$ \\
\hline 1 & Urea & Polycarbonate & 2 & 0.957 & \multirow{2}{*}{0.938} & 0.358 & \multirow{2}{*}{0.375} \\
\hline 2 & Urea & Polycarbonate & 3 & 0.901 & & 0.393 & \\
\hline 3 & Urea & Steel & 2 & 0.865 & \multirow{2}{*}{0.859} & 0.283 & \multirow{2}{*}{0.208} \\
\hline 4 & Urea & Steel & 3 & 0.853 & & 0.132 & \\
\hline 5 & Lentil & Polycarbonate & 2 & 0.855 & \multirow{2}{*}{0.796} & 0.400 & \multirow{2}{*}{0.374} \\
\hline 6 & Lentil & Polycarbonate & 3 & 0.736 & & 0.347 & \\
\hline 7 & Lentil & Steel & 2 & 0.777 & \multirow{2}{*}{0.730} & 0.409 & \multirow{2}{*}{0.373} \\
\hline 8 & Lentil & Steel & 3 & 0.683 & & 0.337 & \\
\hline 9 & Polyethylene & Polycarbonate & 2 & 0.891 & \multirow{2}{*}{0.870} & 0.365 & \multirow{2}{*}{0.294} \\
\hline 10 & Polyethylene & Polycarbonate & 3 & 0.850 & & 0.222 & \\
\hline 11 & Polyethylene & Steel & 2 & 0.802 & \multirow{2}{*}{0.783} & 0.364 & \multirow{2}{*}{0.350} \\
\hline 12 & Polyethylene & Steel & 3 & 0.764 & & 0.335 & \\
\hline
\end{tabular}




\section{CONCLUSIONS}

An experimental device of own design was constructed to measure the coefficient of restitution of small and irregular particles. Three types of particles, two impact materials, and two different launch heights were tested on it. The dependence of the coefficient of restitution with the selected variables was determined by analyzing and studying 120 cases. The tangential values of the coefficient were always higher than the normal values for the studied geometry. In addition, the highest values for the coefficient of restitution's modulus were found for the lower mass particles. The experimental values obtained in this work constitute a starting point to improve the performance of the CFD-DEM simulations of biphasic systems, providing useful information about the energy dissipation and a breakthrough in the study of gassolid flow phenomenon.

\section{REFERENCES}

Colombo, F., K. Seibert, H.G. Espinosa and D.V. Thiel, "Novel methodology for measuring the coefficient of restitution from various types of balls and surfaces," Procedia Engineering, 147, 872-877 (2016).

Crüger, B., V. Salikov, S. Heinrich, S. Antonyuk, V.S. Sutkar, N.G. Deen and J.A.M. Kuipers, "Coefficient of restitution for particles impacting on wet surfaces: An improved experimental approach," Particuology, 24, 1-9 (2016).

Espinosa, H.G., K. Seibert, F. Colombo and D,V. Thiel, "Measuring the court pace rating of tennis courts using low-cost portable devices," Symposium on Sports and Human Dynamics (SHD), Yamagata, Japan (2016).

Fernandez Llana, D., Determinación de parámetros utilizados en las simulaciones D.E.M, Tesis de Máster en Agroingeniería, Universidad Politécnica de Madrid, (2010).

Hastie, D.B., "Experimental measurement of the coefficient of restitution of irregular shaped particles impacting on horizontal surfaces," Chemical Engineering Science, 101, 828-836 (2013).

Kotoky, S., A. Dalal and G. Natarajan, "Effects of specularity and particle-particle restitution coefficients on the hydrodynamic behavior of dispersed gasparticle flows through horizontal channels," $A d$ vanced Powder Technology, 29, 2463-2475, (2018).

Liu, G., F. Yu, H. Lu, S. Wang, L. Pengwei and Z. Hao, "CFD-DEM simulation of liquid-solid fluidized bed with dynamic restitution coefficient," Powder Technology, 304, 186-197 (2016).

Loha, C., H. Chattopadhyay and P.K. Chatterjee, "Effect of coefficient of restitution in Euler-Euler CFD simulation of fluidized-bed hydrodynamics," Particuology, 15, 170-177 (2014).

McNally, W., D. Balzerson, D. Wilson and J. McPhee, "Effect of clubhead inertial properties and driver face geometry on golf ball trajectories," Procedia Engineering, 147, 407-412 (2016).

Takashimizu, Y. and M. Iiyoshi, "New parameter of roundness R: circularity corrected by aspect ratio," Progress in Earth and Planetary Science, 3, 2, 116 (2016).

Received February 20, 2018.

Sent to Guest Editor February 20, 2018.

Accepted February 13, 2019.

Recommended by Guest Editor Patricia M. Hoch 